Vietnam Journal of Mechanics, VAST, Vol. 26, 2004, No.4 (237 - 246)

\title{
USING AN ELECTRO-MECHANICAL SYSTEM TO INVESTIGATE THE INFLUENCE OF THE FATIGUE ON THE ENERGY DISSIPATED PER CYCLE DUE TO THE HYSTERESIS DAMPING
}

\author{
Van Huu Thinh ${ }^{1}$, Nguyen Xuan Hung ${ }^{2}$ \\ 1 University of Technical Education HCM city \\ 2 Institute of Applied Mechanics HCM city
}

\begin{abstract}
The energy dissipated per cycle due to the hysteresis damping is one of the criteria for determining the vibration system. The experimental results reveal that the energy dissipated per cycle increases versus the cycles to fatigue of the rotating-beam steel specimen.
\end{abstract}

\section{Introduction}

To determine the energy dissipated per cycle of the rotating-beam steel specimens, we manufactured and performed the experiments on an electromechanical system as shown in Fig. 1. The electromechanical system consists of a DC motor, a flexible coupling, a revolution counter, bearing housings, a hydraulic jack, and the specimen. In the electromechanical system, the turning on the motor rotates the specimen, and under the load from the hydraulic jack, the specimen is applied a pure bending moment. As the specimen rotates at a point on its outer surface the bending stress varies continuously from maximum tension to maximum compression. Thus this electromechanical system is a type of the rotating-beam fatigue-testing machine. The specimen is a fatigue-testing specimen.

To evaluate the fatigue failure, we can base on one of the following fatigue criteria: the stress-strain criterion, the energy criterion, and the fatigue crack criterion.

In other words, according to vibration of compliant mechanisms we can consider the specimen as a forced vibration system with hysteresis damping. By measuring its vibration signals, we can determine its response and the energy dissipated per cycle. The amount of energy dissipated per cycle equals the area of the hysteresis loop, and the hysteresis loop relates to the fatigue strength of the specimen [4].

This paper presents the basis of theory and the experimental method for determining the change in the energy dissipated per cycle versus the fatigue cycle of the specimens in a rotatingbeam fatigue-testing machine.

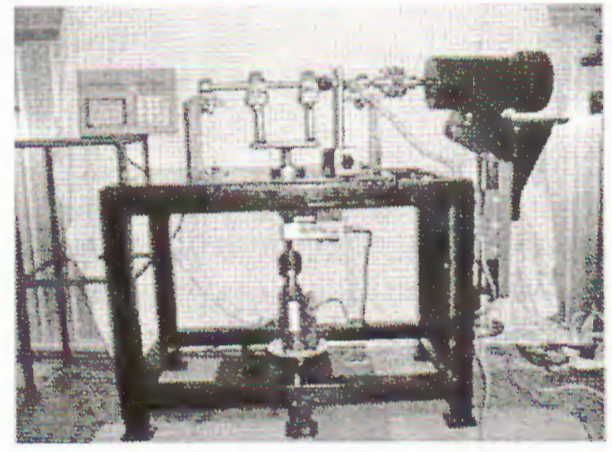

Fig. 1. The fatigue testing machine 


\section{Basis of theory}

\subsection{What is damping}

A structure subjected to oscillating deformation contains a combination of kinetic and potential energy. In the case of real structure, there is also an energy dissipative element as some of the energy is lost per cycle of motion. The amount of energy dissipated is a measure of the structure's inherent damping. In simple terms, damping is the conversion of mechanical energy of a vibrating structure into thermal energy, which is their lost to the structure's environment. There are many kinds of damping, such as Coulomb damping, viscous damping, hysteresis damping (structural damping), air damping, joint damping, magnetic hysteresis damping and piezoelectric damping. The most common kind of damping employed to solve noise and vibration related to fatigue strength is hysteresis damping.

\subsection{Hysteresis damping}

We consider the case of hysteresis damping which is sometimes referred to a structural damping. The influence of this type of damping can be seen in the vibration of solid material, especially metals. In general, solid are not perfectly elastic, when they vibrate, there is an energy dissipated due to internal friction, as result of the relative motion between particles of the solid during deformation. It was observed that there is a phase lag between the applied force $F$ and the displacement $x$, as shown by the hysteresis loop in Fig. 2. It is clear from the figure that the effect of the force does not suddenly disappear when the force is removed.

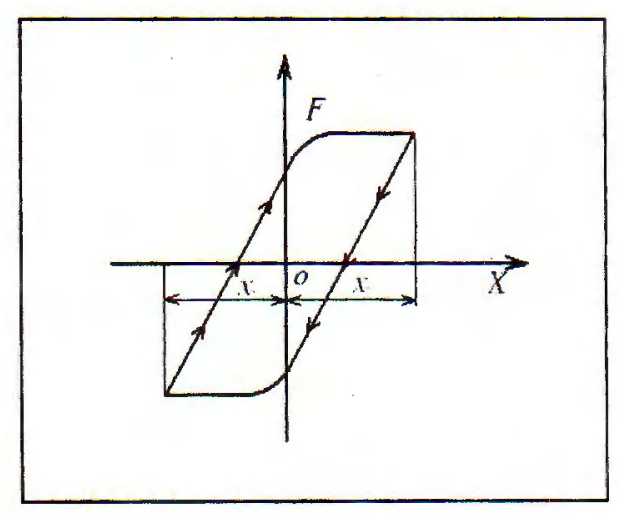

Fig. 2. Hysteresis loop

The energy dissipated per cycle can be obtained as the enclosed area in the hysteresis loop, and can be expressed mathematically using the following integral:

$$
\Delta E=\int F d x .
$$

In system with hysteresis damping, the energy dissipated per cycle of motion is:

$$
\Delta E=\pi k h X^{2},
$$

where $X$ is the amplitude of motion during one cycle, $h$ is the hysteresis damping coefficient, $k$ is the stiffness of spring or system.

In system with viscous damping, the energy dissipated per cycle of motion is:

$$
\Delta E=\pi c_{v} \omega X^{2},
$$

where $c_{v}$ is the viscous damping coefficient, $\omega$ is the forced frequency, $\mathrm{rad} / \mathrm{s}$ 
From Eqs. (2.2) and (2.3), we have the equivalent viscous damping coefficient for the hysteresis damping:

$$
c_{v}=\frac{h k}{\omega} .
$$

The hysteresis damping coefficient cannot be simply specified for a given material. It is a function of geometry as the material. The hysteresis damping is significantly different from viscous damping in that the energy dissipated per cycle for hysteresis damping is independent of the forced frequency, whereas the energy dissipated per cycle increases with the forced frequency for viscous damping.

\subsection{Complex representation}

The use of complex algebra provide an alterative method to the solution of the differential equation governing the forced response of the systems subject to harmonic excitation. It can prove to be less tedious than the use of trigonometric solution.

Recall that if $Q$ is a complex number, it has representation:

$$
Q=Q_{r}+j Q_{i}
$$

where $Q_{r}=\operatorname{Re}(Q)$ is the real part of $Q, Q_{i}=\operatorname{Im}(Q)$ is the imaginary part of $Q$.

The complex number also has the polar form

$$
Q=A e^{j \phi}
$$

where $A$ is the magnitude and $\phi$ is the phase angle of $Q$.

Euler's identity:

$$
e^{j \phi}=\cos \phi+j \sin \phi
$$

leads to

$$
\begin{gathered}
A=\sqrt{Q_{r}^{2}+Q_{i}^{2}}, \\
\phi=\tan ^{-1}\left(\frac{Q_{i}}{Q_{r}}\right) .
\end{gathered}
$$

The equation governing the motion of a system with viscous damping subject to a single frequency sinusoidal excitation can be written as

$$
\ddot{x}+2 \xi \omega_{n} \dot{x}+\omega_{n}^{2} x=\frac{F_{0}}{m} e^{j \omega t},
$$

where $\omega$ is the excitation frequency, $\mathrm{rad} / \mathrm{s} . \omega_{n}$ is the natural frequency, $\mathrm{rad} / \mathrm{s}$ :

$$
\omega_{n}=\sqrt{\frac{k}{m}},
$$

where $k$ is the stiffness of the system, $m$ is the mass of the system, $\mathrm{kg} . F_{o}$ is the magnitude of the excitation $F(t), N . \xi$ is the viscous damping ratio.

$$
2 \xi=\frac{c_{v}}{m} .
$$


A solution of Eq. (2.10) is assumed as

$$
x(t)=H e^{j \omega t},
$$

where $H$ is complex.

By substituting (2.13) into (2.10) leads to

$$
H=\frac{F_{o}}{m\left(\omega_{n}^{2}-\omega^{2}+2 j \xi \omega \omega_{n}\right)} .
$$

Equation (2.14) can be rewritten by using the definition of the frequency ratio $r=\omega / \omega_{n}$ :

$$
\begin{gathered}
H=\frac{F_{o}}{m \omega_{n}\left[\left(1-r^{2}\right)+2 j \xi r\right]} \\
H=\frac{F_{o}\left[\left(1-r^{2}\right)-2 j \xi r\right]}{m \omega_{n}^{2}\left[\left(1-r^{2}\right)^{2}+(2 \xi r)^{2}\right]} .
\end{gathered}
$$

From Eqs. (2.8) and (2.9), $H$ can be written as

$$
H=X e^{-j \phi},
$$

where

$$
X=\frac{F_{o}}{m \omega_{n}^{2}} \frac{1}{\sqrt{\left(1-r^{2}\right)^{2}+(2 \xi r)^{2}}}
$$

a nd

$$
\phi=\tan ^{-1}\left(\frac{2 \xi r}{1-r^{2}}\right) .
$$

The system response is

$$
x(t)=\operatorname{Im}\left(X e^{-j \phi} e^{\omega t}\right)=X \sin (\omega t-\phi) .
$$

From Eqs. (2.4) and (2.10), the equation governing the motion of a system with hysteresis damping subject to a single frequency harmonic excitation can be written as

$$
m \ddot{x}+\frac{h k}{\omega} \dot{x}+k x=F_{o} e^{j \omega t},
$$

where $\omega$ is the excitation frequency, rad/s. Assumption of a solution of the form of Eq. (2.13) leads to

Let

$$
H=\frac{F_{o}}{-m \omega^{2}+k(1+j h)} .
$$

$$
\hat{k}=k(1+j h)
$$

be complex stiffness or complex damping. Thus the forced response of a system with hysteresis damping can be modeled as a system with the complex damping of $\hat{k}=k(1+j h)$. Equation (2.20) becomes

$$
m \ddot{x}+\hat{k} x=F_{o} e^{j \omega t} .
$$


Similar to the calculation above, we have the response of Eq. (2.23):

where

$$
\begin{gathered}
H=\frac{F_{o}}{m \omega^{2}} \frac{\left(1-r^{2}\right)-j h}{\left(1-r^{2}\right)^{2}+h^{2}}, \\
H=X e^{-j \phi}, \\
X=\frac{F_{o}}{m \omega^{2}} \frac{1}{\sqrt{\left(1-r^{2}\right)^{2}+h^{2}}} \\
\phi=\tan ^{-1}\left(\frac{h}{1-r^{2}}\right) .
\end{gathered}
$$

and

Comparing Eqs. (2.24), (2.26) and (2.27) with Eqs. (2.15), (2.17) and (2.18), respectively, it is evident that the steady-state response provided by the hysteresis damping is identical to that with the viscous damping if the hysteresis damping coefficient has the value:

$$
h=2 \xi r
$$

when $r=\frac{\omega}{\omega_{n}}=1$, if the system truly possesses linear viscous damping, the hysteresis loop will be an ellipse as shown by the dashed line in Fig. 3. In this case, the maximum damping force is

$$
F_{D \max }=F_{o}=c_{v} \dot{x}_{\max }=2 \xi m \omega^{2} X
$$

From (2.29), we can determine the viscous damping ratio:

$$
\xi=\frac{F_{o}}{2 m \omega^{2} X}
$$

From Eq. (2.1), the energy dissipated per cycle:

$$
\Delta E=2 \pi \xi \omega \omega_{n} X
$$

where $X$ is determined from Eq. (2.17).

From (2.31), we can also determine the viscous damping ratio $\xi$ and the viscous damping coefficient $c_{v}$ is calculated from (2.12).

If damping is not of linear viscous damping, the hysteresis loop will not be elliptical. It will be of a different shape as illustrated by the solid line in Fig. 3. In this case, the response $x(t)$ will be a distorted harmonic, even though the applied excitation remains a pure harmonic. The energy dissipated per cycle is:

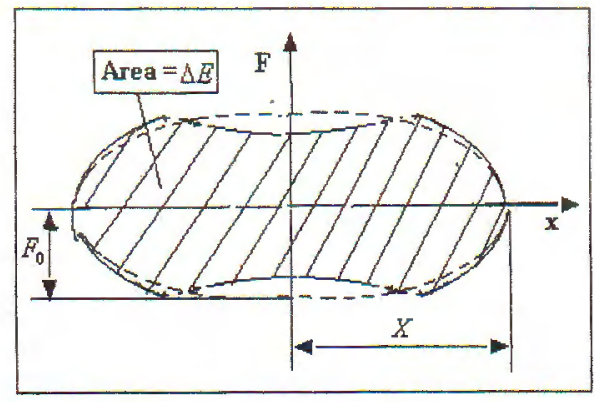

Fig. 3. Actual and equivalent damping energy per cycle

$$
\Delta E=2 \pi \xi_{e q} m \omega^{2} X^{2}
$$

and from (2.32), we can determine the equivalent damping ratio:

$$
\xi_{e q}=\frac{\Delta E}{2 \pi m \omega^{2} X^{2}}
$$


In the case of hysteresis damping, the energy dissipated per cycle is

$$
\Delta E=\pi h m \omega^{2} X^{2},
$$

where $X$ is determined from (2.26). Comparing Eq. (2.34) with Eq. (2.31) reveals that the energy dissipated per cycle for hysteresis damping is independent on the forced frequency, but the energy dissipated per cycle is dependent on the forced frequency for viscous damping.

\subsection{The energy criterion}

According to the fundamentals of fatigue theory, the energy criterion is one of the fatigue criteria.

C.E. Feltner and J.D. Marrow state that the beginning of the fatigue failure will happen if the sum of the energy dissipated equals to the work due to the deformation for the static load.

From Fig. 4, the sum of the energy dissipated after the number of cycles of stress $N$ will be written as

$$
E_{\text {sum }}=2 N \int_{0}^{\Delta \varepsilon} \sigma d \varepsilon,
$$

where $\sigma$ is the stress, $d \varepsilon$ is the strain, $N$ is the cycles of stress

\section{Experimental method}

\subsection{Experiment set up}

The purpose of the experiment is to detect the change in the energy dissipated per cycle due to hysteresis damping of the rotating steel specimen in the rotating beam fatigue testing. Several techniques are used to qualify the level of damping in a specimen or structure:

- Half-Power Bandwidth method.

- Amplification Factor Method.

- Log Decrement Method.

- Hysteresis Loop Method.

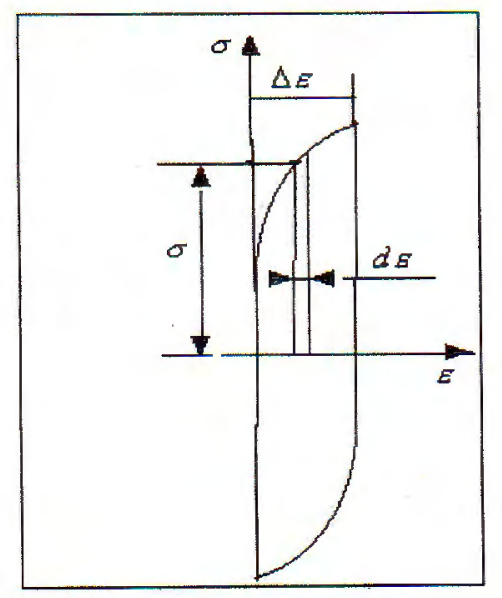

Fig. 4. Hysteresis loop for energy criterion

To determine the energy dissipated per cycle due to hysteresis damping, we use hysteresis loop method for performing the experiment. 
The specimens were made from AISI 1045 steel with the following chemical composition $(\%): \mathrm{C}=0.45, \mathrm{Si}=0.30, \mathrm{Mn}=0.70, \mathrm{~S}=0.04$. The mechanical parameter of this steel is tensile strength $=650 \mathrm{~N} / \mathrm{mm}^{2}$. The shape and dimension of the specimens are given in Fig. 5. The fatigue testing machine is shown in Fig. 1 above.

The experiment conditions for each sample are constant such as the applied force, the speed, and the measurement method. The sample time is $10^{5}$ revolutions of the specimen. The schematic of measuring device system is shown in Fig. 6. It consists of the following devices:

- Load cell + Dynamic force signal converter.

- Revolution counter.

- Proximity probe + Driver + Power supply.

- Amplifier and Filter sets.

- FFT (Fast Fourier Transformer).

- A computer.

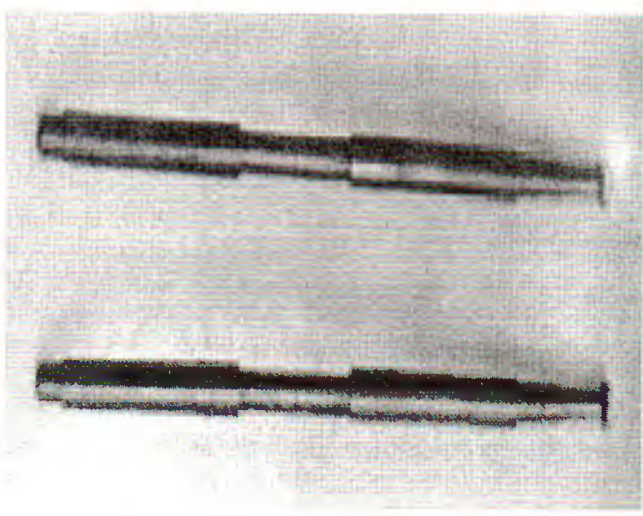

Fig. 5. The specimens

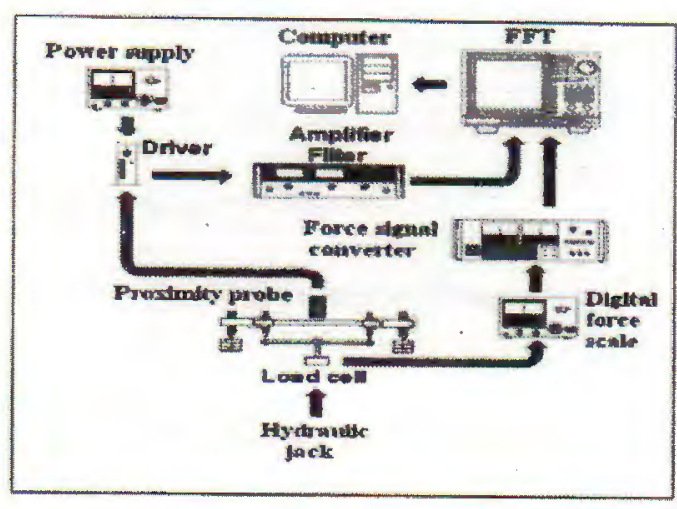

Fig. 6. The schematic of measuring device system

\subsection{The measurement method}

The measured data were displayed on the FFT:

- Channel A: the displacement signal of the rotating specimen (measured by the proximity probe) as shown in Fig. 7.

- Channel B: the dynamic force signal as shown in Fig. 8.

- Combine these two signals, we obtain the hysteresis loop as shown in Fig. 9.

- The measurement data are transmitted to the computer.

- By using the software as shown in Fig. 10, the area of the hysteresis loop is determined. 
Note that the hysteresis loop area $=$ the energy dissipated per cycle.

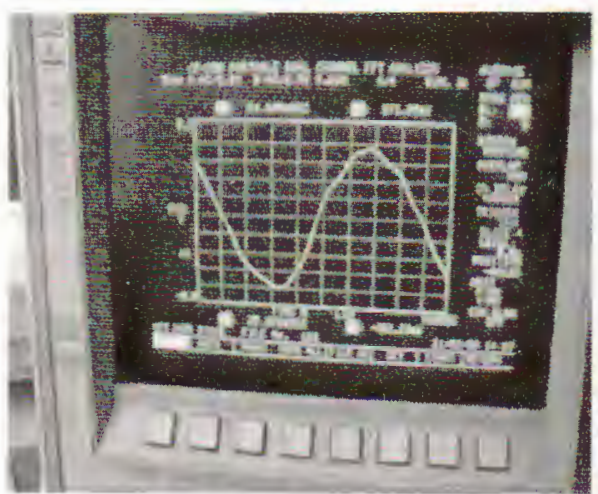

Fig. 7. Displacement signal

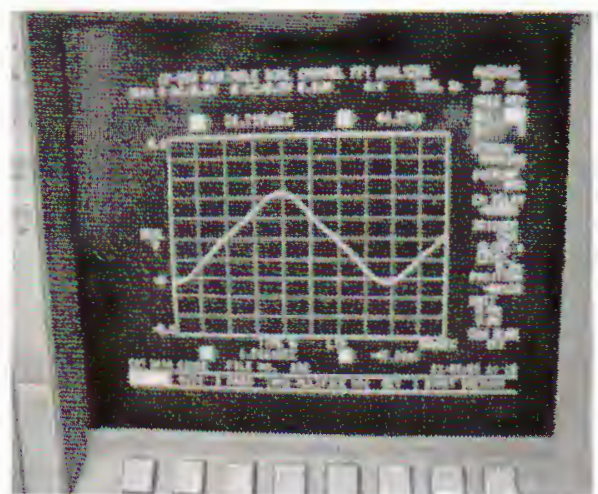

Fig. 8. Force signal

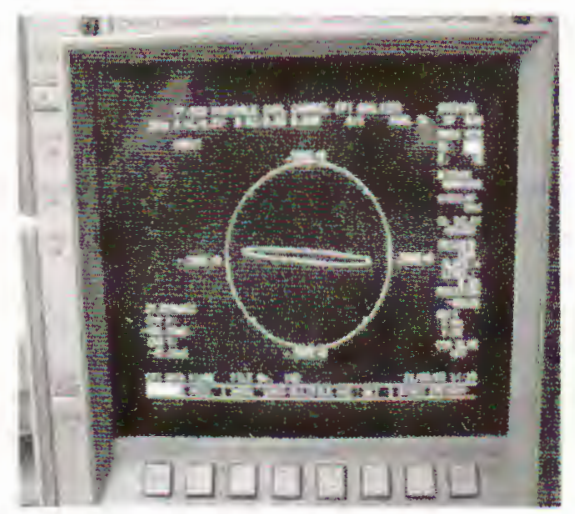

Fig. 9. Hysteresis loop

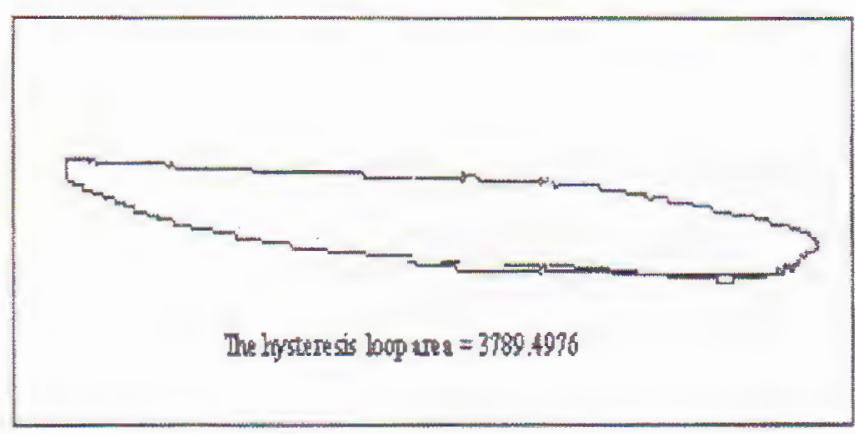

Fig. 10. The hysteresis loop area calculated by software

\subsection{Experimental results}

In order to evaluate the change in the energy dissipated per cycle of the specimen versus the number of the cycle to fatigue, we constructed the plot that shows the relative energy dissipated per cycle $\Delta D$ versus the cycles to fatigue $N$.

$$
\Delta D=\Delta E_{i}-\Delta E_{0}
$$

where $\Delta E_{0}$ is the energy dissipated per cycle spent the number of cycle $N_{0}=0 . \Delta E_{i}$ is the energy dissipated per cycle spent the number of cycle $N_{i}$.

The way in which the energy dissipated per cycle changes depending on the number of fatigue cycles was performed for several specimens applied to the different forces, and the various speed. For illustration we present some experimental results as shown in Fig. 11, and in the following Table 1: 
Table 1

\begin{tabular}{|c|c|c|c|c|c|}
\hline Specimen & Applied force & Speed & Channel A & Channel B & No of cycles \\
\hline 1 & $35 \mathrm{KG}$ & $1000 \mathrm{rpm}$ & $\mathrm{AC} / 1 \mathrm{v}$ & $\mathrm{AC} / 1 \mathrm{v}$ & $25 \times 10^{5}$ \\
\hline 2 & $32 \mathrm{KG}$ & $1100 \mathrm{rpm}$ & $\mathrm{AC} / 0.5 \mathrm{v}$ & $\mathrm{AC} / 0.5 \mathrm{v}$ & $30 \times 10^{5}$ \\
\hline 3 & $32 \mathrm{KG}$ & $1500 \mathrm{rpm}$ & $\mathrm{AC} / 0.5 \mathrm{v}$ & $\mathrm{AC} / 0.5 \mathrm{v}$ & $28 \times 10^{5}$ \\
\hline
\end{tabular}

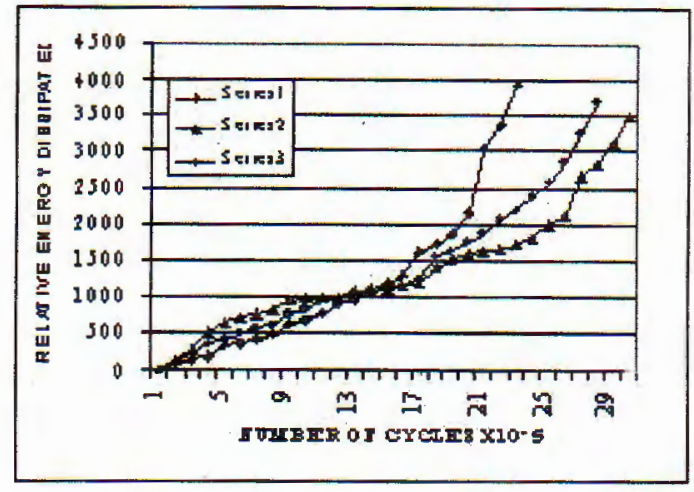

Fig. 11. The change in the energy dissipated per cycle vs number of fatigue cycle

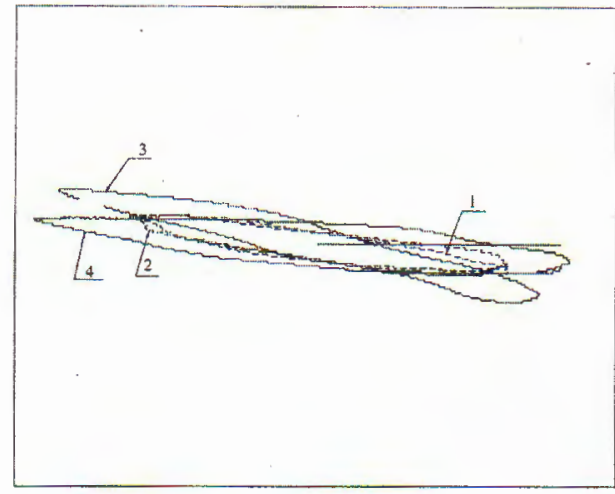

Fig. 12. The change in the hysteresis loop vs number of fatigue cycle

Fig. 11 shows the change in the energy dissipated per cycle versus the number of fatigue cycles of the specimen 1, 2, and 3 labeled series 1, 2, and 3, respectively. Fig. 12 presents the change in the shape and the area of the hysteresis loop depending on the number of fatigue cycles $N$, such as the curve 1: $N=6 \times 10^{5}$ revolutions, $2: N=10 \times 10^{5}$ revolutions, 3: $N=22 \times 10^{5}$ revolutions, $4: N=29 \times 10^{5}$ revolutions of the specimen 2 .

The experimental results reveal that the energy dissipated per cycle increases corresponding to the number of the fatigue cycles, and the hysteresis loop has also been related to the fatigue cycles. This can be explained as the following reason:

- When the specimen rotates there is an energy dissipation due to internal friction, as result of the relative motion between particles of the steel during deformation.

- When the specimen rotates, its stresses and strains are variable with time. Thus, their relation for the hysteresis damping of the specimen should include rates of change. This leads to the energy dissipated per cycle due to the hysteresis damping varies with time.

\section{Conclusions}

This work leads to the following conclusions:

- The energy dissipated per cycle increases depending on the number of the fatigue cycles. It rises sharply from the last time in the life of the specimens. 
- The law's change in the energy dissipated per cycle of the specimen is used for evaluation of the fatigue state, providing the values of the structural damping (hysteresis damping) coefficient, and diagnosing the working life of rotating shaft in a electromechanical system.

This publication is completed with financial support from the National Basic Research Program in Natural Sciences

\section{References}

1. Nguyen Xuan Hung, Van Huu Thinh, The Effect of the Fatigue on elastic Modulus and Damping Coefficient in the Rotating Beam Fatigue Testing, ISDC, Hanoi, 2003.

2. Nguyen Xuan Hung, Dynamics of Structures and compliant Mechanisms, 2003.

3. Shabana A. A., Theory of Vibration, Springer international student edition, 1995.

4. Andrew Dimarogonas, Vibration for Engineers, Prentice-Hall, 1996.

5. Kenneth G. Mc Connell, Vibration Testing, John Wiley \& Sons Inc., 1995.

6. Ray W. Clough, Dynamics. of Structures, McGraw-Hill, Inc., 1993.

7. Graham Kelly S., Fundamentals of mechanical Vibrations, McGraw-Hill, Inc., 2000.

8. Ngô Văn Quyết, Cơ sở lý thuyết mới, Nhà xuất bản Giáo dục, 2000.

Received January 1, 2004

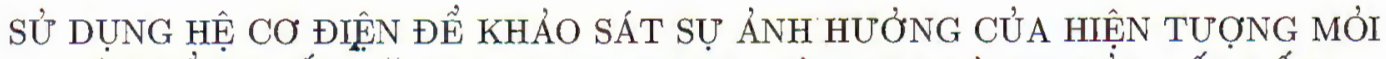 LÊN TỔN THẤT NĂNG LƯỢNG TRONG MỘT CHU KỲ DO CẢN KẾT CẤU}

Tổn thất năng lượng trong một chu kỳ do cản kết cấu là một trong các chỉ tiêu dể tính toán các hệ dao động. Các kết quả nghiên cứu về thực nghiệm cho thấy rằng ảnh hươnng của hiện tượng mỏi làm tăng tồn thất năng lượng trong một chu kỳ theo số chu kỳ phá huý mời cuả mẫu thí nghiệm bằng thép. Ta có thể áp dụng kết quả nghiên cứu này để đánh giá trạng thái mơii, tuổi thọ còn lại và chẩn đoán kỹ thuật đối với mẫu hay trục máy. 